\title{
Parasitic myoma developing at the port site after laparoscopic myomectomy; a case report
}

\author{
Derya Ozturk $^{1 *}$, Orkun Ilgen ${ }^{1}$, Ceren Aydin $^{1}$, Sefa Kurt ${ }^{1}$, Murat Celiloglu \\ ${ }^{1}$ DOKUZ EYLUL UNIVERSITY SCHOOL OF MEDICINE, DEPARTMENT OF OBSTETRICS AND GYNECOLOGY, IZMIR, TURKEY
}

\begin{abstract}
Parasitic myomas occur especially after laparoscopic myomectomy and its incidence increases due to the use of morcellators. Parasitic myomas can rarely occur at the port entry site, being more commonly seen on the intestines, the omentum, the ureter, the bladder, and the diaphragm in different periods after surgery, the average duration being 24 months. In this article, we present the case of parasitic myoma developing secondary to the port site in a 45 -year-old nulliparous woman after the use of laparoscopic myomectomy and morcellation 8 years ago. The patient presented with complaints of pelvic pain and a palpable mass in the left trocar incision line, and an appearance compatible with multiple myomas in the subcutaneous tissue of the left trocar incision line. Besides, myomas were also observed in the mesentery of the rectosigmoid colon and at the uterus in the present study. Numerous and large myomas were observed in the uterus, in the mesentery of the rectosigmoid colon, on the left pelvic peritoneum, and under the skin corresponding to the port entrance. The pathological examination of the myomas excised was reported as leiomyoma. In this article, the diagnosis, the treatment, the possible risk factors, and the complications of myomas were discussed in the light of the literature.
\end{abstract}

Category: Case Presentation
Received: June 09, 2021
Accepted: August 12,2021
Published: November 15,2021
Keywords:
port site myoma, parasitic myomas, laparoscopy,
morcellator
*orresponding author:
Derya Ozturk,
Dokuz Eylul University School of Medicine,
Department of Obstetrics and Gynecology, Izmir,
Turkey, 35340
E-mail: derya.ozturk@deu.edu.tr

\section{Introduction}

Leiomyomas are the most common benign monoclonal tumors of the myometrium among gynecological masses [1]. Myomas are seen in $25 \%$ of the women of reproductive age and are reported in $80 \%$ of post-hysterectomy pathology examinations [2]. Although most women are asymptomatic, myomas may present with dysmenorrhea, menorrhagia, dyspareunia, bladder dysfunction, constipation, and pelvic pain symptoms. Infertility and recurrent abortions may occur due to myoma. Besides, myomas may cause adverse conditions such as premature birth, abnormal placentation, placental abruption, and fetal growth restriction (FGR) [1].

An increase in the uterine size and nodular irregularities due to myoma can be observed upon the physical examination. In myomas located on the cervix, the protruding appearance from the cervix can be observed in the speculum examination. Parasitic fibroids may be asymptomatic or may present symptoms according to their location [3]. The first examination used for diagnosis is ultrasonography (USG) after the anamnesis and the physical examination. A saline infusion sonohysterography can be used in the diagnosis of submucosal and pedunculated intracavitary myomas. Magnetic Resonance Imaging (MRI) provides more useful information in the diagnosis of myomas and parasitic myomas that cannot be diagnosed by USG [4].

The treatment of fibroids depends on whether the patient is symptomatic, the desire for fertility, and age. There are medical, uterine artery embolization, ablation, and surgical treatment options in symptomatic patients [5].

The surgical treatment is an option for patients who do not respond to or fail the medical treatment options. Uterine fibroids are the most common cause of hysterectomies. However, it is not preferred in fertile ages. Uterus-sparing myomectomies can be performed as laparotomy or laparoscopically (L/S) or hysteroscopically $(\mathrm{H} / \mathrm{S})$. The minimally invasive surgical approach (L/S, $\mathrm{H} / \mathrm{S}$ ) has been preferred in the last two decades [6]. In this article, the case of an iatrogenic parasitic myoma in the port site and peritoneal cavity following the use of L / S myomectomy with morcellation is discussed in the light of the literature. 


\section{Case Presentation}

A 45-year-old woman with a diagnosis of myoma uteri, G4P0C4, was admitted to our clinic with chronic pelvic pain and a palpable mass at the level of the old trocar incision in the left suprapubic region. It was revealed from the history of the patient that she had undergone a laparoscopic myomectomy operation in which two myomas with a 6 and 8 -cm diameter were removed 8 years ago and the histopathological examination was compatible with the leiomyoma. In the examination of the case, there was an increase in the size of the uterus due to multiple myomas (approximately 14-week gestational size) and pain in the movements. The adnexal mass was not palpated. In the left lower quadrant of the abdomen, a hard mass, approximately $3-4 \mathrm{~cm}$ in size, was palpated in the trocar incision area of the previous operation. The transvaginal USG performed on the patient revealed numerous myomas with the sizes of $60 \mathrm{~mm}$ on the anterior, $55 \mathrm{~mm}$ posterior, and $45 \mathrm{~mm}$ in the fundus of the uterus. Adnexal pathology was not detected in USG examination. A mass lesion with a peripheral blood supply of $50 \times 30 \times 20 \mathrm{~mm}$ in size was observed in the superficial tissue USG performed for the subcutaneous lesion in the left lower quadrant.

In the MR examination, the uterus was observed enlarged. Numerous myomas deforming and compressing the endometrial cavity, the largest of which was $65 \times 45 \mathrm{~mm}$ in size close to the corpus dorsal part, were reported. A solid lesion of $50 \times 44 \mathrm{~mm}$ in the subcutaneous fat tissue over the external oblique muscles at the level of the left iliac fossa, as well as a 35x54 mm solid lesion compatible with myoma adjacent to the rectosigmoid colon, was reported (Figure 1).

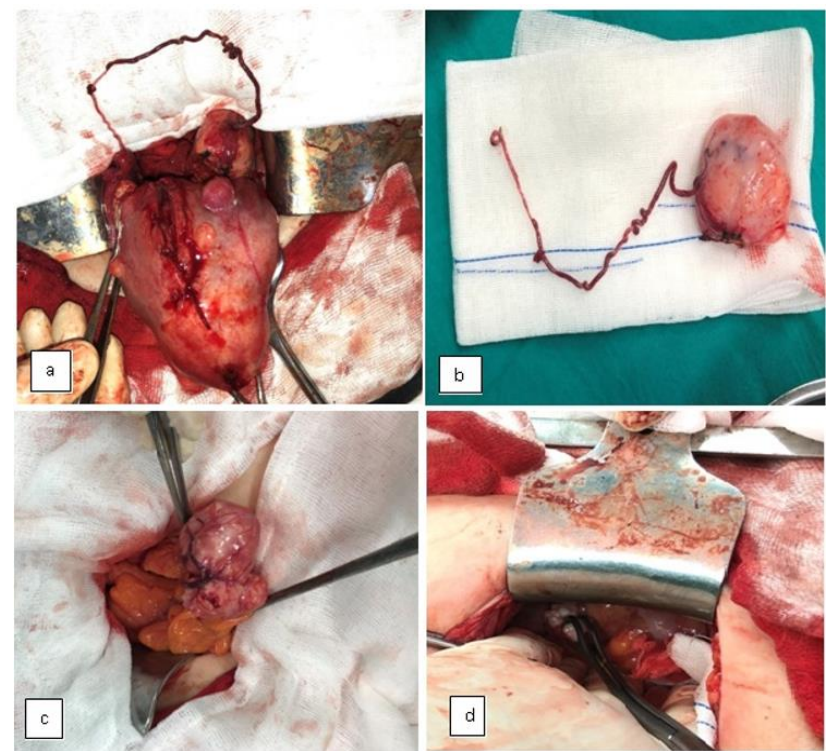

Figure 1. The MRI reveals a mass appearance under the skin with the diameter of $23 \times 35 \mathrm{~mm}$ (a) and other mass in the region of the left iliac fossa adjacent to the sigmoid colon, with the diameter of $35 \times 54 \mathrm{~mm}$ (b).
In the laboratory examinations of the patient, blood biochemistry and tumor markers were detected within normal ranges, except for mild anemia.

A laparotomic myomectomy was performed with a Pfannenstiel incision under general anesthesia. Eight myomas with sizes ranging from $10 \mathrm{~mm}$ to $60 \mathrm{~mm}$ were removed from the uterus. Interestingly, the blood supply of the subserous myoma in the corpus posterior was provided by an isolated neovascular structure (Figure 2). The myoma was removed together with its isolated vascular structure. Subsequently, the masses compatible with the myoma were excised with a diameter of $40 \mathrm{~mm}$ through the left rectosigmoid colon mesentery, $20 \mathrm{~mm}$ from the left pelvic peritoneum, and $30 \mathrm{~mm}$ from the left lower quadrant trocar insertion site (Figure 2 and Figure 3). Both ovaries were observed normally. No complications occurred during the perioperative period. The patient was discharged on the second postoperative day in a good health condition. The pathological results were reported compatible with the leiomyoma.

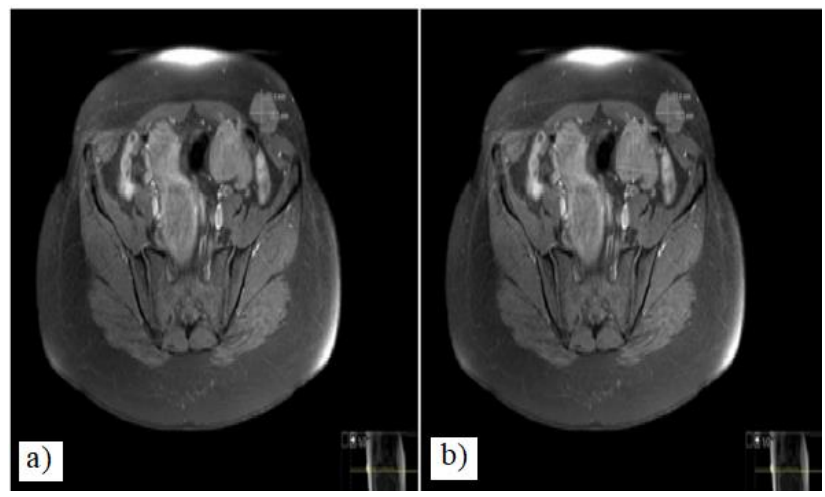

Figure 2. The blood supply of the subsereous myoma in the posterior uterus is provided directly from the right tubal fimbrial end (a). The myoma was excised with a vascular structure (b). A $40 \mathrm{~mm}$ bilobulated mass compatible with the myoma in the left rectosigmoid colon mesentery (c) and another 20-mm mass compatible with the myoma over the left pelvic peritoneum (d) is observed.

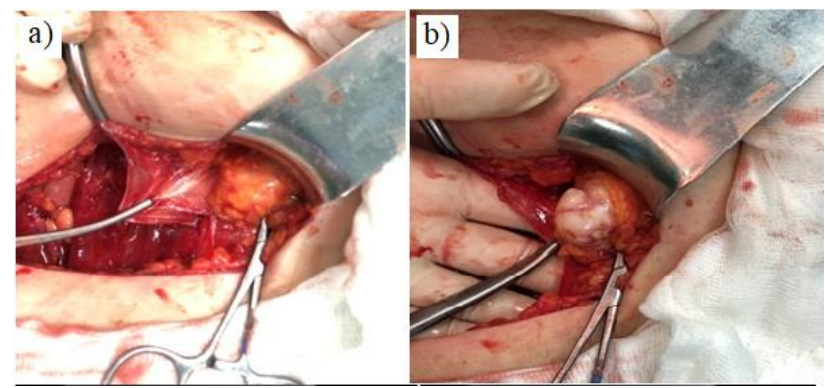

Figure 3. A 30-mm parasitic myoma in the subcutaneous adipose tissue is observed at the left trocar port-site.

\section{Discussion}

Parasitic myomas are rarely seen and mostly pedunculated and localized subserosally. The blood supply is partially or completely separated from the uterus and 
occurs by an alternative vascularization that develops from the omentum or mesenteric vessels [7]. In our case, the subserous myoma in the corpus posterior was vascularized by an isolated arterial structure emerging directly from the right tubal fimbrial end (Figure 2), while the other three myomas had no connection with the uterus, and their blood supply was provided by the omentum and subcutaneous structures where they were completely inoculated. The widespread use of the minimally invasive surgical concept, the increased use of laparoscopic myomectomies, and morcellation may lead to the development of a new parasitic myoma form which can be called iatrogenic parasitic myoma [7].

Studies have shown that the symptoms of iatrogenic myomas are similar to those seen in other types of fibroids. The most common symptoms are pelvic pain, dysmenorrhea, the perception of the mass, and deep dyspareunia. The main complaint of our case is chronic pelvic pain, dysmenorrhea, and palpable mass findings under the skin [7].

USG and MRI can be used for diagnosis. As in our case, parasitic myomas should be considered in the differential diagnosis in cases with laparoscopic myomectomy, a history of morcellation, and extrauterine masses in the echo of myoma. MRI is the most reliable examination in these cases and it can accurately show the location of the tumor in adjacent structures such as the ureter, bladder, and rectum [8]. In our case, MRI was used for diagnosis and the subcutaneous myoma and the myoma on the rectosigmoid colon were revealed, but the parasitic myoma in the left pelvic peritoneum was detected and removed upon the intra-abdominal examination during surgery. In addition, the subcutaneous myoma was also detected by ultrasonography, and it was thought that it may be due to endometriosis since the patient experienced pain.

Although the pathophysiology regarding the cultivation and growth of fibroids is not clear, there is an increase in the extracellular matrix proteins they contain, especially in Type 1 and Type 3 collagen levels. Fibrotic growth factor levels are also irregular in fibroids. Transforming growth factor $\beta$ (TGF- $\beta)$, TGF- $\beta$ receptor, mRNA, and granulocyte-macrophage colony-stimulating factor levels are higher than normal myometrium [9]. Gonadal steroid hormones are known to affect the growth of fibroids. Similarly, it is assumed that prolonged exposure to steroid hormones, such as hormone replacement therapy, may be a risk factor for the development of parasitic fibroids [10]. However, there was no pathology that may need steroid hormone replacement or there was no endogenous steroid hormone increase in the present patient. Parasitic myoma formation after laparoscopic morcellation was first shown by Ostrzenski in 1997 [10]. Studies have reported that the incidence of parasitic myoma has increased due to the use of morcellators [7].
In a review conducted by Pei - Shen Huang et al. between 1998-2011, 19 of the 23 parasitic myoma cases were reported after 2006, suggesting that parasitic myoma formation occurred with the widespread use of minimally invasive surgery. The most important risk factor in the development of iatrogenic parasitic myoma is the history of previous laparoscopic myomectomy and the use of a morcellator [7]. In our case, there was a history of laparoscopic myomectomy operation by using morcellators. In the literature, while the average time between two surgeries ranged from 2 to 108 months, in our case, this period was 106 months.

In the literature review, $78 \%$ of the patients had a laparoscopy with morcellation at the first surgery, and myoma sizes ranged from $8 \mathrm{~mm}$ to $30 \mathrm{~cm}$. In the present case, there was one parasitic myoma of $15 \mathrm{~mm}$ and two parasitic myomas of $30 \mathrm{~mm}$ each. Parasitic myomas settle in various areas in the abdominal cavity such as the intestines, peritoneum, omentum, and port entrance. The myoma can be divided into smaller pieces and removed from the abdomen by morcellation. It is thought that parasitic myomas occur by pouring small myometrial tissues into areas with high blood supply during morcellation in the abdominal cavity [7]. In the present case, the parasitic myoma occurred in the mesentery above the sigmoid colon, pelvic peritoneum, and port entry site. These areas should be carefully controlled at the end of the surgery. To avoid this complication, it is recommended to do the morcellation inside the bag and perform plenty of peritoneal washing procedures [3].

After the laparoscopic morcellation, besides the development of the parasitic myoma, malignant cell spread can also be seen. The FDA issued a warning stating that there was one case of death resulting from the spread of malignant cells following an unsuspected leiomyosarcoma morcellation [11].

\section{Conclusions}

Iatrogenic parasitic myomas, similar to port site involvement after laparoscopic malignancy or endometriosis surgery, are a condition associated with the widespread use of laparoscopic myomectomy and morcellation, and presents with complaints of palpable mass and pain at the port site. Conventional measures such as morcellation in the endobag, washing the abdomen with abundant saline solution, careful observation, and the evacuation of $\mathrm{CO} 2$ before the ports are removed prevent the development of port-site and intra-abdominal parasitic myoma. To conclude, it should be kept in mind that parasitic myoma involvement in the laparoscopic port-site may occur after myomectomy, as in malignancy and endometriosis. 


\section{Author Contributions Statement}

D.O: manuscript writing, data collection; S.K: review of the manuscript, data collection; O.I: manuscript writing; M.C: review of the manuscript, supervision, C.A: manuscript writing.

\section{Conflict of interest disclosure}

There are no known conflicts of interest in the publication of this article. The manuscript was read and approved by all authors.

\section{Compliance with ethical standards}

Any aspect of the work covered in this manuscript has been conducted with the ethical approval of all relevant bodies and that such approvals are acknowledged within the manuscript. This report followed the guidelines to be HIPAA compliant and permission was obtained from the patient to publish identifiable photographs. The study adhered to the tenets of the Declaration of Helsinki. The written informed consent was obtained from the patient for the publication of this case report and any accompanying images.

\section{References}

1. Munro MG, Critchley HO, Broder MS, Fraser IS; FIGO Working Group on Menstrual Disorders. FIGO classification system (PALM-COEIN) for causes of abnormal uterine bleeding in nongravid women of reproductive age. Int J Gynaecol Obstet. 2011 Apr;113(1):3-13. doi: 10.1016/j.ijgo.2010.11.011

2. Sparic R, Mirkovic L, Malvasi A, Tinelli A. Epidemiology of Uterine Myomas: A Review. Int J Fertil Steril. 2016 Jan-Mar;9(4):424-35. doi: $10.22074 /$ ijfs.2015.4599
3. Donnez J, Dolmans MM. Uterine fibroid management: from the present to the future. Hum Reprod Update. 2016;22(6):665-686. doi: 10.1093/humupd/dmw023

4. Testa AC, Di Legge A, Bonatti M, Manfredi R, Scambia G. Imaging techniques for evaluation of uterine myomas. Best Pract Res Clin Obstet Gynaecol. 2016;34:37-53. doi: 10.1016/j.bpobgyn.2015.11.014

5. Laughlin-Tommaso SK. Alternatives to Hysterectomy: Management of Uterine Fibroids. Obstet Gynecol Clin North Am. 2016 Sep;43(3):397-413. doi: 10.1016/j.ogc.2016.04.001

6. Owen C, Armstrong AY. Clinical management of leiomyoma. Obstet Gynecol Clin North Am. 2015 Mar;42(1):67-85. doi: 10.1016/j.ogc.2014.09.009

7. Huang PS, Chang WC, Huang SC. Iatrogenic parasitic myoma: a case report and review of the literature. Taiwan J Obstet Gynecol. 2014 Sep;53(3):392-6. doi: 10.1016/j.tjog.2013.11.007

8. Paul PG, Shintre H, Mehta S, Gulati G, Paul G, Mannur S. Parasitic Myomas: An Unusual Risk after Morcellation. Gynecol Minim Invasive Ther. 2018 JulSep;7(3):124-126. doi: 10.4103/GMIT.GMIT_36_18

9. Stewart EA, Laughlin-Tommaso SK, Catherino $\mathrm{WH}$, Lalitkumar S, Gupta D, Vollenhoven B. Uterine fibroids. Nat Rev Dis Primers. 2016 Jun 23;2:16043. doi: 10.1038/nrdp.2016.43

10. Van der Meulen JF, Pijnenborg JM, Boomsma CM, Verberg MF, Geomini PM, Bongers MY. Parasitic myoma after laparoscopic morcellation: a systematic review of the literature. BJOG. 2016 Jan;123(1):69-75. doi: 10.1111/1471-0528.13541

11. Vilos GA, Allaire C, Laberge PY, Leyland N; SPECIAL CONTRIBUTORS. The management of uterine leiomyomas. J Obstet Gynaecol Can. 2015; 37(2):157-178. doi: 10.1016/S1701-2163(15)30338-8 